\title{
马铃薯产量组分的基因型与环境互作及稳定性
}

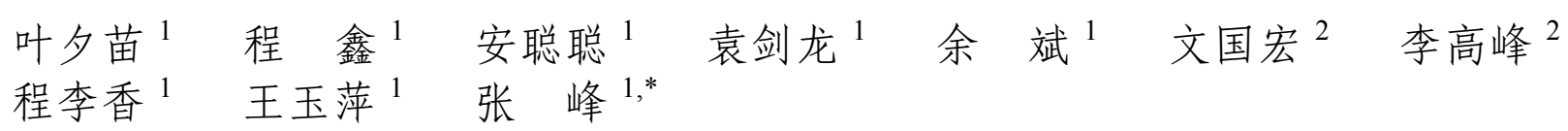

${ }^{1}$ 甘肃农业大学 / 甘肃省干旱生境作物学国家重点实验室培育基地 / 甘肃省遗传改良与种质创新重点实验室, 甘肃兰州 730070 ;

2 甘肃省农业科学院 / 马铃薯研究所, 甘肃兰州 730070

摘 要: 本研究主要探究基因型和基因型与环境互作(genotype + genotypes and environment interactions, GGE)双标图 在马铃薯育种中的应用。综合评价马铃薯品系产量性状在不同环境中的丰产性、稳定性和适应性, 篮选出适应不同 生态环境的产量性状优良品系。同时评价各试点的区分力和代表性, 为试点的选择提供依据。2015 年和 2016 年在甘 肃安定区鲁家沟镇、安定区内官镇、渭源县五竹镇 3 个试点种植国际马铃薯中心引进的 101 份高代品系和对照青薯 9 号。收获后记录小区产量、小区大薯产量、小区小薯产量、单株产量、单株大薯产量、单株小薯产量、单株结薯 数、单株大薯数、单株小薯数; 采用联合方差和 GGE 双标图对产量性状进行基因型与环境互作分析。方差分析表明, 除小区小薯产量在基因型与环境互作效应中无显著差异外, 其他产量组分在基因型效应、环境效应和互作效应中均 呈现极显著差异 $(P<0.01)$ 。小区产量、小区大薯产量、小区小薯产量、单株产量、单株大薯产量、单株结薯数环境效 应平方和占总方差平方和最大; 单株小薯产量、单株大薯数和单株小薯数的基因型与环境互作效应平方和占总方差 平方和最大。GGE 分析结果表明, 适应性最强的品系在鲁家沟试点是 G86; 在五竹镇试点是 G65; 在内官镇试点是 G86。参试品系中丰产品系有 G86、G116、G124；稳产品系有 G124、G125、G10；高产稳产品系有 G86、G116、G124、 青薯 9 号。单株大薯数高的品系有 G45、G86、G67, 稳定性好的品系有 G67、G116、G51, 对照青薯 9 号的单株大 薯产量不稳定。综合鉴别力和代表性的强弱, 依次为鲁家沟镇 2016 年、鲁家沟镇 2015 年、五竹镇 2015 年、五竹镇 2016 年、内官镇 2015 年、内官镇 2016 年。GGE 模型能够直观地展现多年多点品系试验结果, 并客观评价参试品系 的丰产性、稳定性和适应性, 同时可以对试点的代表性和区分力进行评价。以 GGE 模型综合评价, 高产稳产品系有 G116、G124、G125、G122、青薯 9 号; 高产不稳定的品系有 G86、G10、G121、G106、G107、G72。最理想的生 态区试点是鲁家沟镇, 对品种的鉴别力最强的试点是五竹镇。

关键词: 产量组分; GGE 双标图; 多年多点; 试点评价

\section{Genotype $\times$ environment interaction and stability of yield components for po- tato lines}

YE Xi-Miao ${ }^{1}$, CHENG Xin ${ }^{1}$, AN Cong-Cong ${ }^{1}$, YUAN Jian-Long ${ }^{1}$, YU Bin ${ }^{1}$, WEN Guo-Hong ${ }^{2}$, LI Gao-Feng ${ }^{2}$, CHENG Li-Xiang ${ }^{1}$, WANG Yu-Ping ${ }^{1}$, and ZHANG Feng ${ }^{1, *}$

\footnotetext{
${ }^{1}$ Gansu Agricultural University / Gansu Provincial Key Laboratory of Aridland Crop Science / Gansu Key Laboratory of Crop Improvement \& Germplasm Enhancement, Lanzhou 730070, Gansu, China; ${ }^{2}$ Gansu Acadamy of Agricultural Sciences / Potato Insititute, Lanzhou 730070, Gansu, China
}

\footnotetext{
本研究由国家重点研发计划项目(2017YFD0101905), 国家自然科学基金项目(31471433), 甘肃省高等学校协同创新团队项目 (2018C-17)和甘肃省科技重大专项计划项目(17ZD2NA016)资助。

This study was supported by the National Key R\&D Program of China (2017YFD0101905), the National Natural Science Foundation of China (31471433), Gansu High Educational Scientific Special Project (2018C-17), and Gansu Province Science and Technology Major Special Projects (17ZD2NA016).

* 通信作者(Corresponding author): 张峰, E-mail: zhangf@gsau.edu.cn

第一作者联系方式: E-mail: yeximiaogs@163.com

Received (收稿日期): 2019-06-21; Accepted (接受日期): 2019-09-26; Published online (网络出版日期): 2019-10-11.

URL: http://kns.cnki.net/kcms/detail/11.1809.s.20191011.1215.004.html
} 


\begin{abstract}
This study mainly focused on the application of GGE (genotype + genotypes and environment interactions) biplot in potato breeding, to evaluate the productivity, stability and adaptability of yield traits of potato lines in different environments comprehensively, and screen out the excellent lines adapted to different mage-environments. The representativeness and discriminating ability of each test-environment were also evaluated, providing a basis for the selection of test-environment. A total of 101 advanced lines from International Potato Center (CIP) and potato variety Qingshu 9 were planted in Neiguan Town, Lujiagou Town and Wuzhu Town of Gansu province in 2015 and 2016 to measure the plot yield, plot yield of large-sized tubers, plot yield of small-sized tubers, yield per plant, large-sized tuber yield per plant, small-sized tuber yield per plant, tuber number per plant, large-sized tuber number per plant and small-sized tuber number per plant. The genotype and environment interactions were analyzed by the combined analysis of variance and GGE biplot. Except the plot yield of small-sized tubers had no significant difference in genotype and environment interactions effect, all the other yield components had significant differences $(P<0.01)$ in genotype effect, environmental effect and genotype and environment interaction effect. The square sum of environmental effect on the plot yield, plot yield of large-sized tubers, plot yield of small-sized tubers, yield per plant, large-sized tuber yield per plant, tuber number per plant, and the square sum of genotype and environment interaction effect on the plot yield of small-sized tubers, the large-sized tuber number per plant, and the small-sized tuber number per plant were worth the largest in the square sum of total variance. The most adaptable lines in Lujiagou Town were G86, in Wuzhu Town G65, in Neiguan Town G86. The high-yield lines were G86, G116, and G124; the stable-yield lines were G124, G125, and G10; the high-yielding and stable lines were G86, G116, G124, and Qingshu 9. The lines with more large-sized tuber number per plant were G45, G86, and G67, and the lines with good stability were G67, G116, and G51. The variety Qingshu 9 did not have stable large-sized tuber yield per plant. According to the comprehensive discrimination and representativeness, the order of test-environments were Lujiagou Town in 2016, Lujiagou Town in 2015, Wuzhu Town in 2015, Wuzhu Town in 2016, Neiguan Town in 2015, and Neiguan Town in 2016. GGE model can intuitively display the results in the genotype-location-year framework, and objectively evaluate the productivity, stability and adaptability of tested lines, as well as the representativeness and discriminating ability of test-environment. According to the comprehensive evaluation of GGE model, the high-yielding and stable lines were G116, G124, G125, G122, and Qingshu 9, and the high-yielding and unstable lines were G86, G10, G121, G106, G107, and G72. The most ideal mage-environment is Lujiagou Town, and Wuzhu Town is the test-environment with the strongest discriminating ability for varieties identification.
\end{abstract}

Keywords: yield component; GGE biplot; multi-years and sites; pilot evaluation

马铃薯是主要的宜粮宜菜作物。为了选育出多 适和多用途类型的品种, 需要对各类种质资源和选 育出的品系进行产量、抗性、品质等方面的系统评 价，并获得在不同生态区的适应性、丰产性和稳定 性的基础数据, 综合评价和篮选出产量性状优良的 品系 ${ }^{[1-2]}$ 。同时评价各试点的区分力和代表性, 为引 进资源和品系筛选的理想试点提供选择依据。不同 的基因型在不同环境会表现出性状差异, 这些差异 是由基因型、环境以及基因型与环境互作效应共同 作用的结果 ${ }^{[3]}$ 。前期的研究表明, 作物多数性状都会 表现出不同环境表型差异的互作现象 ${ }^{[4-7]}$ 。对于产量 性状稳定性, 早期的研究方法是平均产量的变异系 数说明基因型的稳定性, 这种方法只能说明基因型 效应的差异, 不能说明环境效应以及基因型与环境 互作效应。利用线性回归对品种的稳定性研究也不 能体现出品种对环境的适应性特点。应用方差分析 评价品种的适应性不能分析环境效应和互作效应的 关系 ${ }^{[8-11]}$ 。将互作效应分解为基因型分量和环境分量 的 AMMI 模型, 虽然可以用双标图直观展现基因型 与环境互作关系。但 AMMI 模型只考虑互作效应, 没有将互作效应和基因型主效相结合 ${ }^{[12]}$ 。基因型 $(G)$ 和基因型与环境互作 $(\mathrm{GE})$ 联合效应的 GGE 模型综
合考虑 $\mathrm{G}$ 和 $\mathrm{GE}$ 效应，可以同时对基因型和环境评 价 ${ }^{[13]}$ 。前期 GGE 模型已经在水稻、小麦、玉米等作 物的产量、抗性、品质的基因型、基因型和环境互 作中进行了研究 ${ }^{[14-18]}$ 。对于马铃薯这样的同源四倍 体作物，大量农艺性状由多个等位位点决定，基 因主效性不显著的作物中，基于基因型和基因型 与环境互作联合效应的研究还没有广泛开展和验 证 $^{[19-21]}$ 。本研究进行 2 年 3 点试验, 拟通过 GGE 模 型分析 101 份引进种质资源的高代品系产量及产量 组分性状，阐述基因型与环境互作效应对评价马铃 薯块茎产量和产量组分的实际效果, 综合产量组分 筛选出优良的品系, 同时评价 GGE 模型在区分试验 点的代表性和鉴别力方面的作用。

\section{1 材料与方法}

\section{1 试验材料}

引自国际马铃薯中心的 101 份高代品系(表 1), 其高代品系来源于 5 类不同群体, 包括 Population A、B3C1、LBHT1 (晚熟、抗晚疫病、高淀粉, 适宜 油炸加工、耐热)、Intermediate LT-LB (中早熟、菜 用、抗晚疫病)和 LTVR (耐热、抗病毒病、菜用、具 低海拔高温适应性)(https://cipotato.org/catalogue), 
表 1102 份引进国际马铃薯中心高代品系

Table 1102 introduced potato advanced lines from CIP

\begin{tabular}{|c|c|c|c|c|c|}
\hline $\begin{array}{c}\text { 品系编号 } \\
\text { Line number }\end{array}$ & $\begin{array}{l}\text { CIP 编号 } \\
\text { CIP entry }\end{array}$ & $\begin{array}{c}\text { 品系编号 } \\
\text { Line number }\end{array}$ & $\begin{array}{l}\text { CIP 编号 } \\
\text { CIP entry }\end{array}$ & $\begin{array}{c}\text { 品系编号 } \\
\text { Line number }\end{array}$ & $\begin{array}{l}\text { CIP 编号 } \\
\text { CIP entry }\end{array}$ \\
\hline G1 & CIP 381381.13 & G45 & CIP 385561.124 & G92 & CIP 397099.6 \\
\hline G3 & CIP 391583.25 & G46 & CIP 388676.1 & G93 & CIP 397100.9 \\
\hline G4 & CIP 392617.54 & G48 & CIP 390478.9 & G94 & CIP 397196.3 \\
\hline G5 & CIP 392634.52 & G49 & CIP 391207.2 & G95 & CIP 397196.8 \\
\hline G8 & CIP 393227.66 & G50 & CIP 391382.18 & G96 & CIP 397197.9 \\
\hline G9 & CIP 393228.67 & G51 & CIP 392781.1 & G98 & CIP 388611.22 \\
\hline G10 & CIP 393371.164 & G52 & CIP 392797.22 (青薯 9 号 Qingshu 9) & G99 & CIP 388615.22 \\
\hline G11 & CIP 391004.18 & G53 & CIP 392822.3 & G100 & CIP 389468.3 \\
\hline G12 & CIP 392657.171 & G54 & CIP 392973.48 & G101 & CIP 390637.1 \\
\hline G13 & CIP 393280.64 & G56 & CIP 394034.65 & G102 & CIP 391180.6 \\
\hline G14 & CIP 391047.34 & G57 & CIP 394034.7 & G104 & CIP 391724.1 \\
\hline G15 & CIP 391058.175 & G58 & CIP 394579.36 & G105 & CIP 392032.2 \\
\hline G16 & CIP 393085.5 & G59 & CIP 394600.52 & G106 & CIP 392740.4 \\
\hline G17 & CIP 398192.213 & G61 & CIP 394613.139 & G107 & CIP 392745.7 \\
\hline G18 & CIP 398098.119 & G62 & CIP 394613.32 & G108 & CIP 392759.1 \\
\hline G19 & CIP 398098.203 & G63 & CIP 394614.117 & G109 & CIP 393613.2 \\
\hline G21 & CIP 398180.289 & G64 & CIP 394881.8 & G110 & CIP 393615.6 \\
\hline G22 & CIP 398180.292 & G65 & CIP 395186.6 & G112 & CIP 397030.31 \\
\hline G23 & CIP 398180.612 & G67 & CIP 395195.7 & G113 & CIP 397035.26 \\
\hline G25 & CIP 398203.509 & G68 & CIP 395196.4 & G114 & CIP 302428.20 \\
\hline G27 & CIP 398208.33 & G70 & CIP 395432.51 & G115 & CIP 302476.108 \\
\hline G30 & CIP 301024.14 & G71 & CIP 395434.1 & G116 & CIP 302499.30 \\
\hline G31 & CIP 301029.18 & G72 & CIP 395436.8 & G118 & CIP 304350.100 \\
\hline G32 & CIP 301040.63 & G74 & CIP 396311.1 & G119 & CIP 304350.118 \\
\hline G33 & CIP 300046.22 & G77 & CIP 397014.2 & G120 & CIP 304350.95 \\
\hline G35 & CIP 300054.29 & G79 & CIP 397029.21 & G121 & CIP 304371.67 \\
\hline G36 & CIP 300056.33 & G81 & CIP 397039.51 & G122 & CIP 304383.41 \\
\hline G37 & CIP 300063.4 & G82 & CIP 397044.25 & G123 & CIP 304383.80 \\
\hline G39 & CIP 300072.1 & G84 & CIP 397065.2 & G124 & CIP 304387.39 \\
\hline G40 & CIP 300093.14 & G85 & CIP 397067.2 & G125 & CIP 304405.47 \\
\hline G41 & CIP 300099.22 & G86 & CIP 397069.5 & G127 & CIP 397077.16 \\
\hline G42 & CIP 300101.11 & G87 & CIP 397073.15 & G128 & CIP 391919.3 \\
\hline G43 & CIP 379706.27 & G88 & CIP 397078.12 & G129 & CIP 391930.1 \\
\hline G44 & CIP 385499.11 & G91 & CIP 397098.12 & G131 & CIP 394906.6 \\
\hline
\end{tabular}

其中 G52 (CIP 392797.22)为试验对照品种青薯 9 号。

\section{2 试验设计}

2015 年和 2016 年分别在渭源县五竹镇、安定区 内官镇和安定区鲁家沟镇 3 个试点(表 2)采用随机区组 设计, 播种 $50 \mathrm{~g}$ 切块种薯, 设每个品种(系) 3 个小区, 单垄双行种植, 行距 $40 \mathrm{~cm}$, 株距 $25 \mathrm{~cm}$, 小区面积为 $1.1 \mathrm{~m} \times 2.5 \mathrm{~m}$ 。所有试验不施肥, 无灌溉, 苗期进行覆 土固苗(10 15 cm 覆土深度), 其余栽培和田间管理措
施均按统一方法，保持各地块一致。每年 4 月 25 日至 30 日播种, 9 月 15 日至 25 日收获。收获期测定产量和 产量组分。参照《马铃薯试验研究方法》 ${ }^{[22]}$ 统计小区 产量、小区大薯产量、小区小薯产量; 单株产量、单 株大薯产量、单株小薯产量; 单株结薯数、单株大薯 数、单株小薯数(块茎大小以 $75 \mathrm{~g}$ 为界限划分)。

\section{3 试验点平均降水和气温状况} 2015-2016 年五竹镇年平均降水量 $437.6 \mathrm{~mm}$, 
年平均气温 $6.4^{\circ} \mathrm{C}$, 内官镇年平均降水量 $529 \mathrm{~mm}$, 年平均气温 $9.9^{\circ} \mathrm{C}$, 鲁家沟镇年平均降水量 $253 \mathrm{~mm}$, 年平均气温 $9.1^{\circ} \mathrm{C}$ 。3 个试验点的平均降水和气温状
况(表 2)均高于历史平均值, 其中内官镇的年平均降 水量比历史平均值增加了 $35 \%$ 。产量组分分析中, 环 境为试验地点和年份的组合。

表 2 试点环境

Table 2 Basic information of tested locations

\begin{tabular}{|c|c|c|c|c|c|c|}
\hline $\begin{array}{c}\text { 试点 } \\
\text { Location }\end{array}$ & $\begin{array}{c}\text { 试点编号 } \\
\text { Location code }\end{array}$ & $\begin{array}{c}\text { 海拔 } \\
\text { Altitude } \\
\text { (m) }\end{array}$ & $\begin{array}{c}\text { 年降水量 } \\
\text { Annual precipitation } \\
(\mathrm{mm})\end{array}$ & $\begin{array}{c}\text { 年日照时数 } \\
\text { Annual sunshine } \\
\text { (h) }\end{array}$ & $\begin{array}{c}\text { 年平均温度 } \\
\text { Mean annual } \\
\text { temperature }\left({ }^{\circ} \mathrm{C}\right)\end{array}$ & $\begin{array}{c}\text { 无霜期 } \\
\text { Frostless } \\
\text { period (d) }\end{array}$ \\
\hline 五竹镇 Wuzhuzhen & WZ & 2450 & 540 & 2462 & 3.5 & 145 \\
\hline 内官镇 Neiguanzhen & NG & 2080 & 390 & 2050 & 6.2 & 141 \\
\hline 鲁家沟镇 Lujiagouzhen & LJG & 1898 & 220 & 2780 & 6.3 & 160 \\
\hline
\end{tabular}

\section{4 数据分析}

采用 Microsoft Excel 2016 和 SPSS 19.0 软件统 计分析数据。采用 GGEbiplot 8.0 软件处理基因型和 环境互作模型数据。

\section{5 稳定性分析}

GGE 模型被称为环境中心化的主成分分析，在 原始数据中减去环境的平均值后将基因型在互作效 应中进行奇异值分解 ${ }^{[23]}$ 。

$$
Y_{g e r}=\mu+\beta_{e}+\sum \lambda_{n} \gamma_{g n} \delta_{e n}+\rho_{g e}+\varepsilon_{g e r}
$$

其中, $Y_{g e r}$ 是基因型 $g$ 在环境 $e$ 中的第 $r$ 个重复的产 量或者其他性状值。 $\mu$ 是总体平均值, $\beta_{e}$ 是环境 $e$ 的均值。 $\lambda_{n}$ 是第 $n$ 个主成分的奇异值, $\gamma_{g n}$ 是基因型 $g$ 在第 $n$ 个特征向量得分, $\delta_{e n}$ 是环境 $e$ 在第 $n$ 个特 征向量得分, $\rho_{g e}$ 基因型 $g$ 在环境 $e$ 的残差, $\varepsilon_{g e r}$ 是总 体误差。

GGE 双标图是基于前 2 个主成分 PC1 和 PC2, 因此公式(1)可转化如下:

$$
Y_{\text {ger }}=\mu+\beta_{e}+\lambda_{1} \gamma_{g 1} \delta_{e 1}+\lambda_{1} \gamma_{g 2} \delta_{e 2}+\rho_{g e}+\varepsilon_{\text {ger }}
$$

其中, $\lambda_{1}$ 是第 1 个主成分的奇异值, $\gamma_{g 1}$ 是基因型 $g$ 在
第 1 个特征向量得分。 $\delta_{e 1}$ 是环境 $e$ 在第 1 个特征向 量得分, 以此类推。

\section{2 结果与分析}

\section{1 方差分析}

高代品系产量性状及产量组分在基因型、环境 和基因型 $\times$ 环境互作效应均极显著，而小区小薯产 量在基因型与环境互作方面差异不显著(表 3)。产量 性状组分中单株小薯产量、单株小薯数的互作效应 平方和占总方差平方和比例分别为 $39.1 \%$ 和 $35.0 \%$, 基因型效应占比分别为 $18.9 \% 、 19.6 \%$, 环境效应占 比分别为 $2.3 \%$ 和 $2.1 \%$; 小区产量、小区大薯产量、 小区小薯产量、单株产量、单株大薯产量和单株结 薯数环境效应的平方和占比分别为 $34.0 \% 、 28.0 \%$ 、 $18.2 \% 、 25.9 \% 、 24.7 \%$ 和 $32.8 \%$, 互作效应占比分别 为 $33.4 \% 、 27.6 \% 、 22.8 \% 、 12.1 \% 、 22.8 \% 、 22.90 \%$ 和 $26.7 \%$, 基因型效应占比分别为 $17.2 \%$ 、3.4\%、 $8.6 \%$ 、15.5\%、16.0\%和 $10.6 \%$ 。单株大薯数互作效 应平方和占比为 $33.9 \%$, 环境效应占比 $19.5 \%$, 基因 型效应占比为 $3.7 \%$ 。

\section{表 3 马铃薯产量性状方差分析}

\begin{tabular}{|c|c|c|c|c|c|c|}
\hline $\begin{array}{l}\text { 性状 } \\
\text { Trait }\end{array}$ & $\begin{array}{c}\text { 变异来源 } \\
\text { Source of variation }\end{array}$ & $\begin{array}{c}\text { 自由度 } \\
\mathrm{df}\end{array}$ & $\begin{array}{c}\text { 平方和 } \\
\text { Sum of square }\end{array}$ & $\begin{array}{c}\text { 均方 } \\
\text { Mean squares }\end{array}$ & $\begin{array}{c}F \text { 检验 } \\
F \text {-test }\end{array}$ & $\begin{array}{c}\text { 显著性 } \\
\text { Significance }\end{array}$ \\
\hline 小区产量 & 基因型 G & 101 & 6142.832 & 60.8201 & 26.97 & $<0.001$ \\
\hline \multirow[t]{4}{*}{ Plot yield } & 环境 E & 5 & 12098.734 & 1228.566 & 276.57 & $<0.001$ \\
\hline & 互作 $\mathrm{G} \times \mathrm{E}$ & 505 & 11907.641 & 23.579 & 5.31 & $<0.001$ \\
\hline & 残差 Residual & 1224 & 5437.244 & 4.442 & & \\
\hline & 总变异 Total & 1835 & 35586.452 & & & \\
\hline 小区大薯产量 & 基因型 G & 101 & 933.319 & 9.2408 & 8.29 & $<0.001$ \\
\hline \multirow{2}{*}{$\begin{array}{l}\text { Plot yield of large-sized } \\
\text { tuber }\end{array}$} & 环境 E & 5 & 7671.285 & 1534.257 & 20.39 & $<0.001$ \\
\hline & 互作 $\mathrm{G} \times \mathrm{E}$ & 505 & 7585.699 & 15.021 & 1.64 & $<0.001$ \\
\hline
\end{tabular}

Table 3 Analysis of variance for yield traits in potato lines 
(续表 3)

\begin{tabular}{|c|c|c|c|c|c|c|}
\hline $\begin{array}{l}\text { 性状 } \\
\text { Trait }\end{array}$ & $\begin{array}{c}\text { 变异来源 } \\
\text { Source of variation }\end{array}$ & $\begin{array}{c}\text { 自由度 } \\
\mathrm{df}\end{array}$ & $\begin{array}{c}\text { 平方和 } \\
\text { Sum of square }\end{array}$ & $\begin{array}{c}\text { 均方 } \\
\text { Mean squares }\end{array}$ & $\begin{array}{l}F \text { 检验 } \\
F \text {-test }\end{array}$ & $\begin{array}{c}\text { 显著性 } \\
\text { Significance }\end{array}$ \\
\hline \multirow{6}{*}{$\begin{array}{c}\text { 小区小薯产量 } \\
\text { Plot yield of } \\
\text { small-sized tuber }\end{array}$} & 残差 Residual & 1224 & 11207.720 & 9.157 & & \\
\hline & 总变异 Total & 1835 & 27398.024 & & & \\
\hline & 基因型 $\mathrm{G}$ & 101 & 1323.103 & 13.100 & 1.71 & $<0.001$ \\
\hline & 环境 E & 5 & 2806.794 & 561.359 & 73.31 & $<0.001$ \\
\hline & 互作 $\mathrm{G} \times \mathrm{E}$ & 505 & 1867.107 & 3.697 & 0.48 & 1 \\
\hline & 残差 Residual & 1224 & 9372.213 & 7.657 & & \\
\hline \multirow{5}{*}{ Yield of tuber per plant } & 总变异 Total & 1835 & 15369.218 & & & \\
\hline & 基因型 $\mathrm{G}$ & 101 & 119.526 & 1.1834 & 5.30 & $<0.001$ \\
\hline & 环境 E & 5 & 198.688 & 39.7376 & 177.82 & $<0.001$ \\
\hline & 互作 $\mathrm{G} \times \mathrm{E}$ & 505 & 174.808 & 0.3462 & 1.55 & $<0.001$ \\
\hline & 残差 Residual & 1224 & 273.529 & 0.2235 & & \\
\hline \multirow{5}{*}{$\begin{array}{l}\text { Yield of large-sized } \\
\text { tubers per plant }\end{array}$} & 总变异 Total & 1835 & 766.550 & & & \\
\hline & 基因型 $\mathrm{G}$ & 101 & 119.390 & 1.1821 & 5.38 & $<0.001$ \\
\hline & 环境 E & 5 & 183.742 & 36.7485 & 167.16 & $<0.001$ \\
\hline & 互作 $\mathrm{G} \times \mathrm{E}$ & 505 & 169.916 & 0.3365 & 1.53 & $<0.001$ \\
\hline & 残差 Residual & 1224 & 269.091 & 0.2198 & & \\
\hline \multirow{4}{*}{$\begin{array}{l}\text { Yield of small-sized } \\
\text { tubers per plant }\end{array}$} & 总变异 Total & 1835 & 742.139 & & & \\
\hline & 基因型 G & 101 & 2.882 & 0.0285 & 5.79 & $<0.001$ \\
\hline & 环境 E & 5 & 0.352 & 0.0704 & 14.28 & $<0.001$ \\
\hline & 互作 $\mathrm{G} \times \mathrm{E}$ & 505 & 5.948 & 0.0118 & 2.39 & $<0.001$ \\
\hline \multirow{6}{*}{$\begin{array}{l}\text { Number of tuber per } \\
\text { plant }\end{array}$} & 残差 Residual & 1224 & 6.029 & 0.0049 & & \\
\hline & 总变异 Total & 1835 & 15.211 & & & \\
\hline & 基因型 G & 101 & 2978.502 & 29.490 & 4.32 & $<0.001$ \\
\hline & 环境 E & 5 & 9208.894 & 1841.779 & 270.03 & $<0.001$ \\
\hline & 互作 $\mathrm{G} \times \mathrm{E}$ & 505 & 7497.026 & 14.846 & 2.18 & $<0.001$ \\
\hline & 残差 Residual & 1224 & 8348.500 & 6.821 & & \\
\hline \multirow{4}{*}{$\begin{array}{l}\text { Number of large-sized } \\
\text { tubers per plant }\end{array}$} & 总变异 Total & 1835 & 28032.922 & & & \\
\hline & 基因型 G & 101 & 475.476 & 4.708 & 5.54 & $<0.001$ \\
\hline & 环境 E & 5 & 2510.065 & 502.014 & 21.21 & $<0.001$ \\
\hline & 互作 $\mathrm{G} \times \mathrm{E}$ & 505 & 4349.834 & 8.614 & 1.92 & $<0.001$ \\
\hline \multirow{7}{*}{$\begin{array}{c}\text { 单株小薯数 } \\
\text { Number of small-sized } \\
\text { tubers per plant }\end{array}$} & 残差 Residual & 1224 & 5487.667 & 4.483 & & \\
\hline & 总变异 Total & 1835 & 12822.982 & & & \\
\hline & 基因型 G & 101 & 2434.490 & 24.104 & 5.51 & $<0.001$ \\
\hline & 环境 E & 5 & 270.209 & 54.042 & 12.35 & $<0.001$ \\
\hline & 互作 $\mathrm{G} \times \mathrm{E}$ & 505 & 4347.791 & 8.609 & 1.97 & $<0.001$ \\
\hline & 残差 Residual & 1224 & 5354.333 & 4.374 & & \\
\hline & 总变异 Total & 1835 & 12406.824 & & & \\
\hline
\end{tabular}

G: genotype; E: environment; $\mathrm{G} \times \mathrm{E}$ : genotype $\times$ environment.

\section{2 产量性状适应性 GGE 双标图分析}

GGE 双标图的多边形图(which-won-where)根据 基因型-环境的互作效应对试点分组(图 1-A F), 多 边形由同一方向上距离原点最远的基因型连接而成, 所有基因型都包括在内。通过从原点到多边形各边
垂线将双标图分成若干扇区，试点落在扇区中不同 区域。GGE 多边形图中基因型小区产量、小区大薯 产量、小区小薯产量、单株产量、单株大薯产量和 单株小薯产量 $\mathrm{G}+\mathrm{GE}$ 占比分别为 $74.9 \% 、 77.7 \%$ 、 $65.4 \% 、 69.8 \% 、 70.1 \%$ 和 $68.0 \%$ (图 1-A F)。 
试点所在区域中，“角顶” 基因型是产量最高 的基因型。小区产量 GGE 双标图中, G86、G27、G43、 G53、G41、G109、G1、G65 连接成多边形, 8 条射 线将多边形分为 2 个扇区, 第一个扇区位于射线 1 和 2 之间, 试点内官镇和鲁家沟镇落在扇区 1 内,
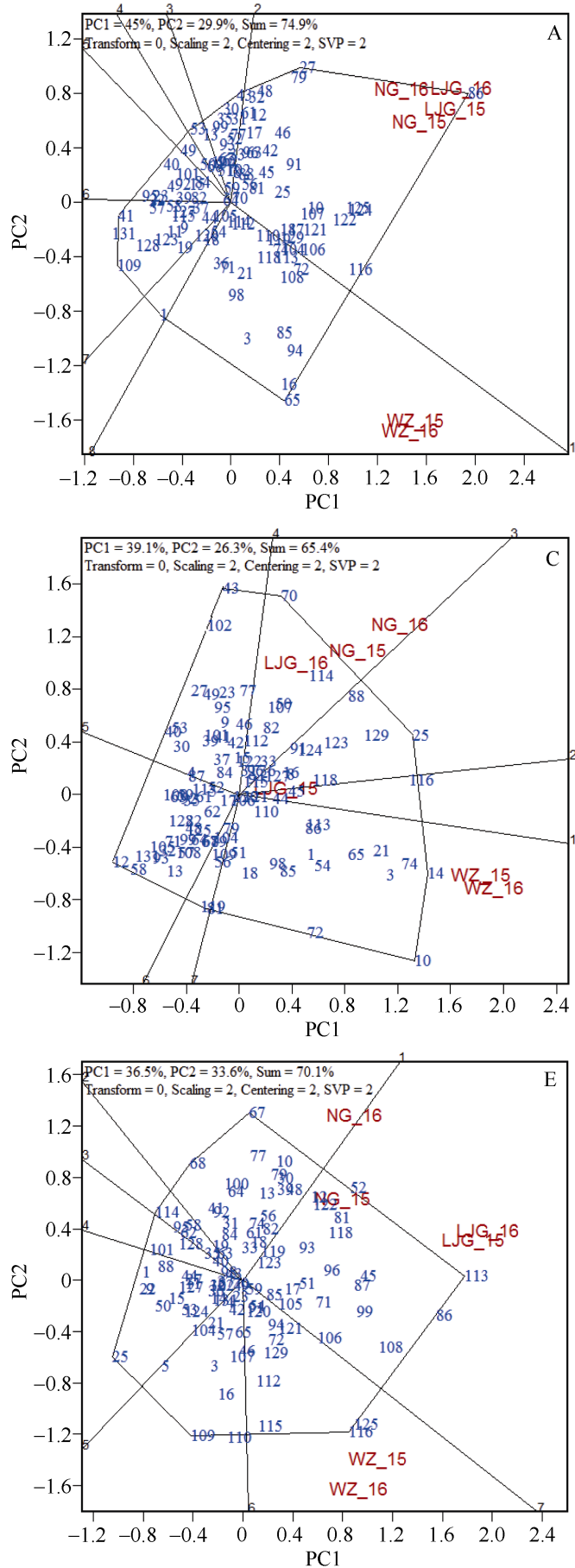

G86 产量最高; 第二个扇区位于射线 1 和 8 之间，试 点五竹镇落在扇区 2 内, G65 产量最高。无试点的扇 区中，“角顶”基因型在所有试点中表现不佳。没 有试点落于以基因型 G43、G53、G41、G109、G1 为角顶的区内, 这 5 个基因型在所有试点中小区产
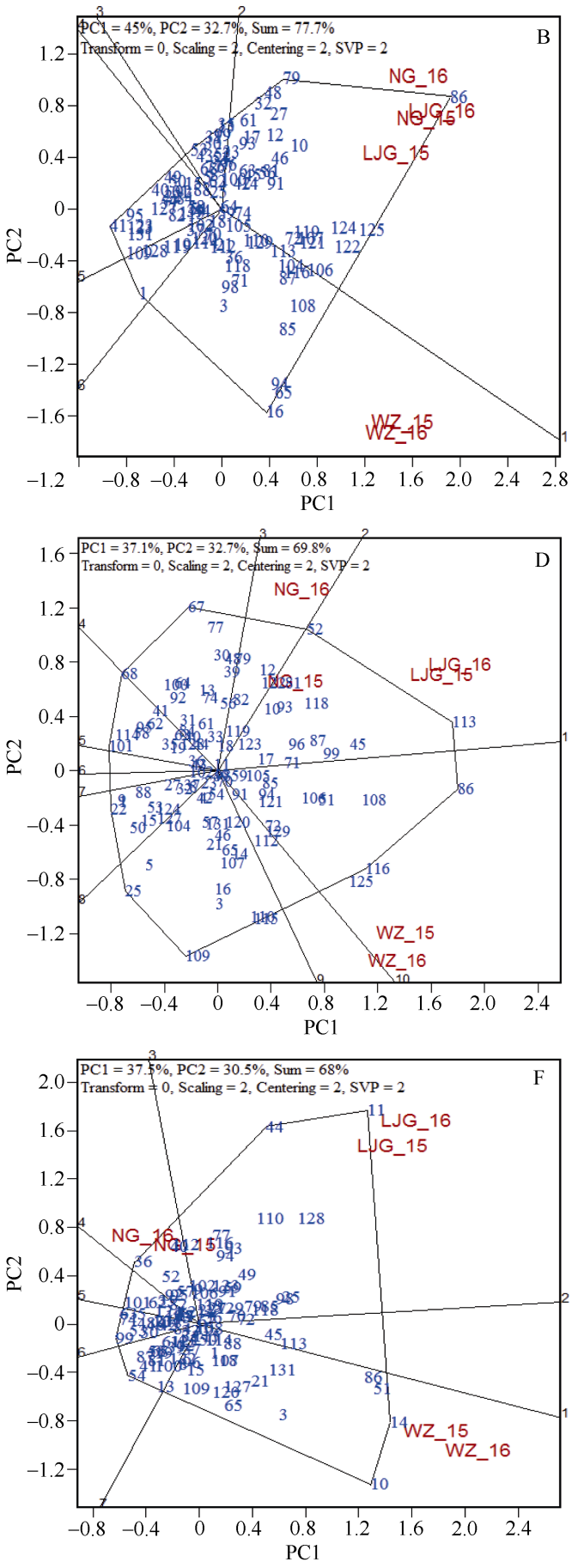

图 1 产量性状适应性 GGE 分析

Fig. 1 Analysis of yield traits adaptability by the GGE biplot

A：小区产量; B：小区大薯产量; C：小区小薯产量; D：单株产量; E：单株大薯产量; F：单株小薯产量。

A: the yield of plot; B: the plot yield of large-sized tuber; C: the plot yield of small-sized tuber; D: the yield per plant; E: the yield of large-sized tubers per plant; F: the yield of small-sized tubers per plant. 
量表现不佳(图 1-A)。“角顶”基因型不同，连接成的 多边形不同，产量相关性状双标图被分成不同的扇 区，相同试点中优势基因型不同(图 1-A F)。小区大 薯产量 GGE 双标图中, G86、G79、G53、G41、G1、 G16 连接成的多边形分为 2 个扇区, 试点内官镇和 鲁家沟镇中 G86 产量最高, 试点五竹镇中 G16 产量 最高(图 1-B); 小区小薯产量 GGE 双标图中 G14、 G25、G70、G43、G12、G109、G10 连接成的多边 形分为 2 个扇区, 试点内官镇和鲁家沟镇中 G70 产 量最高, 试点五竹镇中 G10 的产量最高(图 1-C); 单 株产量 GGE 双标图中 G86、G113、青薯 9 号、G67、 G68、G101、G22、G25、G109、G116 连接成的多 边形分为 3 个扇区, 试点鲁家沟镇中 G113 产量最高, 试点五竹镇中 G86 的产量最高, 试点内官镇 2016 年 青薯 9 号产量最高(图 1-D); 单株大薯产量 GGE 双 标图中 G113、G67、G68、G114、G25、G109、G116 连接成的多边形分为 3 个扇区, 试点鲁家沟镇和内 官镇 2015 年中 G113 产量最高, 试点 2016 年内官镇 中 G67 产量最高, 鲁家沟镇和内官镇 2015、2016 年 中青薯 9 号产量均在多边形边线上, 属于 2 个区域 的高产品种, 试点五竹镇中 G116 产量最高(图 1-E); 单株小薯产量 GGE 双标图中 G11、G44、G36、G99、 G54、G10、G14 连接成的多边形分为 3 个扇区, 试 点鲁家沟镇中 G11 产量最高, 试点内官镇中 G36 产 量最高, 试点五竹镇中 G10 产量最高(图 1-F)。

\section{3 产量丰产性和稳定性 GGE 双标图分析}

理想基因型应具有最高的产量均值和最高的稳 定性。产量丰产性和稳定性 GGE 双标图(Mean vs. stability view)呈现的是基因型的高产性与稳定性。 单向箭头从原点指向平均环境作为平均环境轴 (average environment axis, AEA), 箭头所指方向代 表较高的产量平均值。垂直于 AEA 轴的双向箭头的 直线为纵轴, 作为平均环境坐标 (average environment coordination, AEC), 2 个箭头指向较不稳定产 量平均值, 越接近于 $\mathrm{AEA}$ 轴, 基因型稳定性越好, $\mathrm{GE}$ 效应越小(图 2-A F)。

小区产量 GGE 双标图中, 各试点中平均产量依 次为 G86、G124、G116 等, G131 平均产量最低; 各 试点中产量稳定性依次以 G124、G82、G64 等最高, G65 稳定性最差; 各试点中高产不稳定的基因型有 G86、G94、G27 等; 各试点中低产稳定的基因型有 G64、G82、G39 等; 各试点中高产稳定基因型有 G124、G125、G122 (图 2-A)。小区大薯产量 GGE
双标图中, 各试点中平均产量依次为 G86、G125、 G122 等, G41 平均产量最低; 各试点中产量稳定性 依次为 G57、G64、G58 等; 各试点中高产不稳定 的基因型有 G86、G85、G65 等; 各试点中低产稳 定的基因型有 G64、G44、G58 等; 各试点中高产 稳定的基因型有 G125、G124、G121（图 2-B)。小 区小薯产量 GGE 双标图中, 各试点中平均产量依 次为 $\mathrm{G} 25 、 \mathrm{G} 116 、 \mathrm{G} 14$ 等; 各试点中产量稳定性依 次为 G62、G120、G123 等; 各试点中高产不稳定 的基因型有 G14、G74、G70 等; 各试点中低产稳 定的基因型有 G17、G62、G22 等; 各试点中高产 稳定的基因型有 G123、G124、G91 (图 2-C)。青薯 9 号在小区产量、小区大薯产量和小区小薯产量上 均呈现较高的稳定性。

单株产量 GGE 双标图中, 各试点中平均产量依 次为 G113、G86、G108 等; 各试点中产量稳定性依 次为 G99、G102、G17 等; 各试点中高产不稳定的 基因型有 G86、G108、G116 等; 各试点中低产稳 定的基因型有 G12、G98、G49 等; 各试点中高产 稳定的基因型有 G113、G45、G99 (图 2-D)。单株 大薯产量 GGE 双标图中, 各试点中平均产量依次 为 $\mathrm{G} 113 、 \mathrm{G} 86 、 \mathrm{G} 108$ 等; 各试点中产量稳定性依 次为 G51、G102、G1 等; 各试点中高产不稳定的 基因型有 G125、G116, 青薯 9 号在单株大薯产量 中同样呈现出高产不稳定; 各试点中低产稳定的基 因型有 G98、G37、G44 等; 各试点中高产稳定的 基因型有 G45、G87、G96 (图 2-E)。单株小薯产量 GGE 双标图中, 各试点中平均产量依次为 G11、 G44、G128 等; 各试点中产量稳定性依次为 G58、 G32、G87 等; 各试点中高产不稳定的基因型有 G44、G51、G14 等; 各试点中低产稳定的基因型有 G12、67、G108 等; 各试点中高产稳定的基因型有 G25、G98、G85 (图 2-F)。

\section{4 试点鉴别力和代表性 GGE 双标图分析}

试点评价 GGE 双标图中接近原点的小圆圈代 表平均环境, 通过原点和平均环境的单向直线所指 同心圆的中心代表理想试点。双标图中的同心圆有 助于判断平均环境到 AEA、AEC 和双标图原点的距 离。理想试点应该是有鉴别力和代表性的。理想试 点是所有试点中向量最长, 且具有绝对代表性的虚 拟试点。试点向量越长, 对基因型鉴别力越强; 试点 与 AEA 的夹角越小, 对理想环境代表性越强。2 个 试点向量间夹角的余弦值与它们之间的遗传相关系 

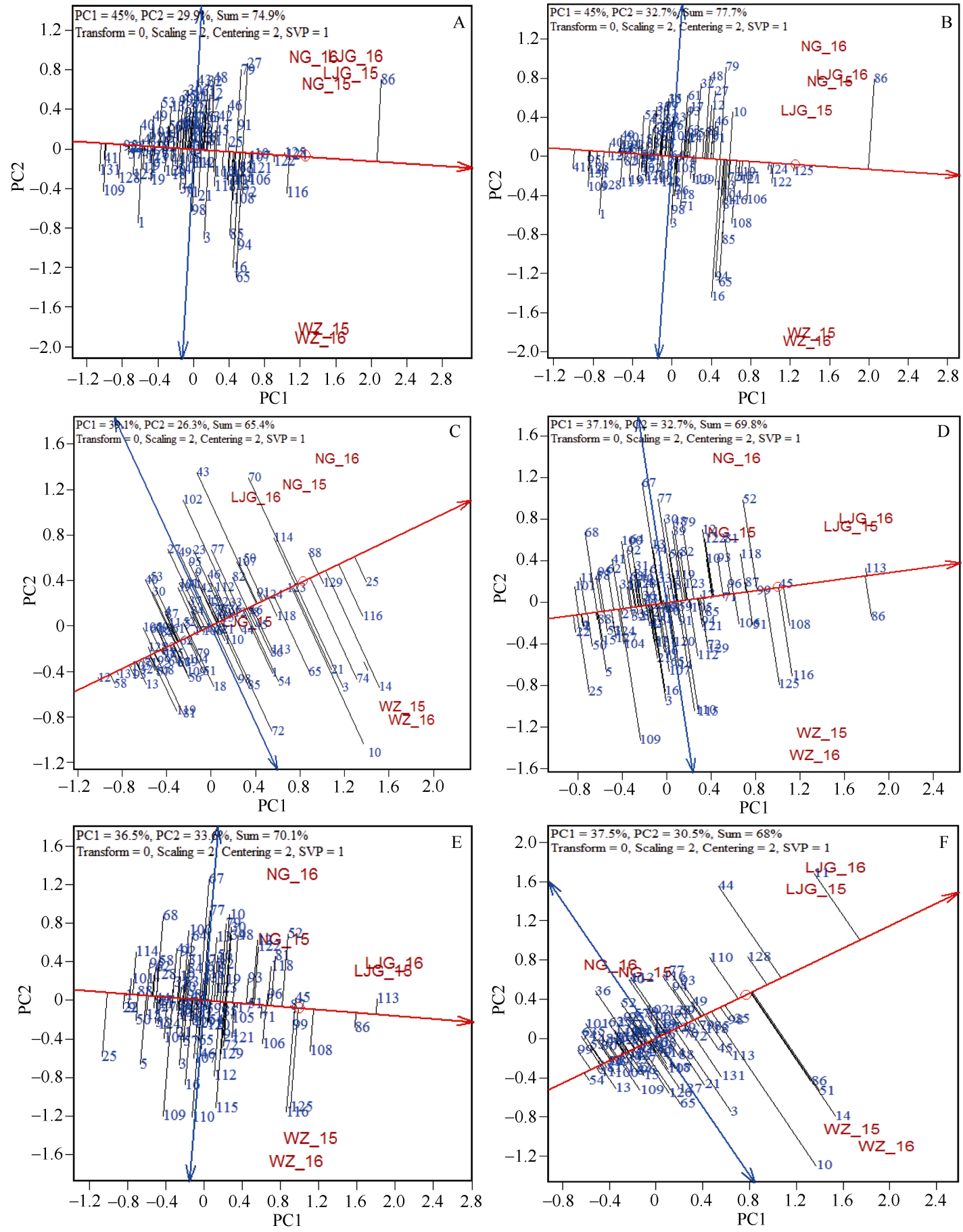

图 2 丰产性和稳定性 GGE 分析

Fig. 2 Mean yield performance and stability of potato lines by the GGE biplot

A：小区产量; B：小区大薯产量; C：小区小薯产量; D：单株产量; E：单株大薯产量; F：单株小薯产量。

A: the yield of plot; B: the plot yield of large-sized tuber; C: the plot yield of small-sized tuber; D: the yield per plant; E: the yield of large-sized tubers per plant; F: the yield of small-sized tubers per plant.

数近似, 夹角小于 90 度表示正相关, 对品种排序相 似，大于 90 度表示负相关，对品种排序相反，等于 90 度, 两试点不相关。

在试点评价 GGE 双标图中, 3 个试点被分为 2 个差异明显的品种生态区，五竹镇属一个品种生态
区，内官镇和鲁家沟镇属另外一个相同生态区。同 一试点年份间鉴别力和代表性存在差别，3 个试点 2016 年较 2015 年对基因型鉴别力更强, 而 2015 年 环境代表性较 2016 年更强(图 3-A F)。试点鲁家沟 镇和内官镇在 2015 年和 2016 年份对产量组分中小 
区产量、小区大薯产量、单株产量和单株大薯产量 鉴别最接近理想试点; 五竹镇是 2015 年和 2016 年 份中鉴别力最强的试点(图 3-A, B, D, E)。试点鲁家 沟镇 2016 年份对小区小薯产量最具鉴别力, 鲁家沟 镇 2015 最具代表性。综合鉴别力和代表性, 试点排 序依次为鲁家沟镇 2015、鲁家沟镇 2016、五竹镇
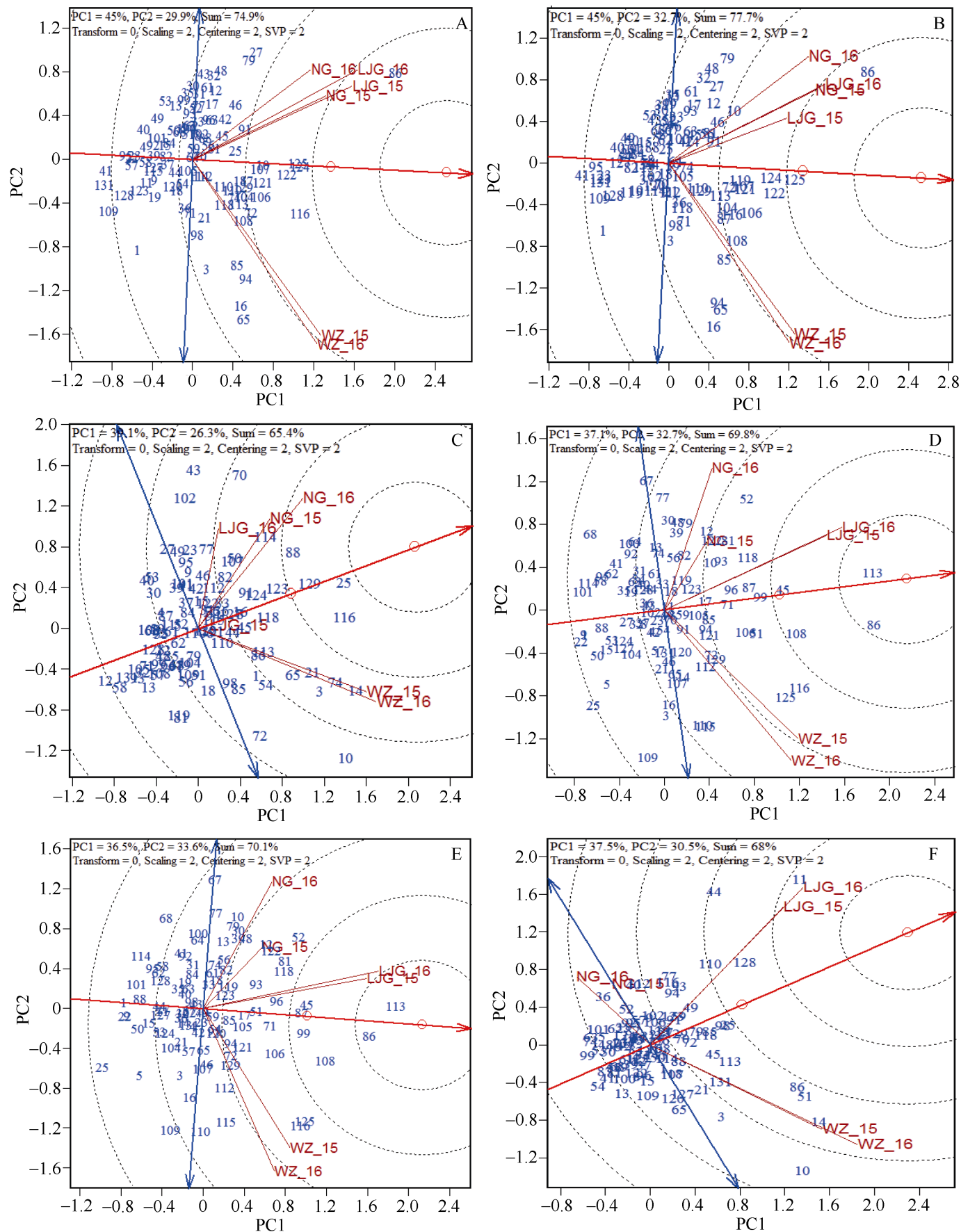

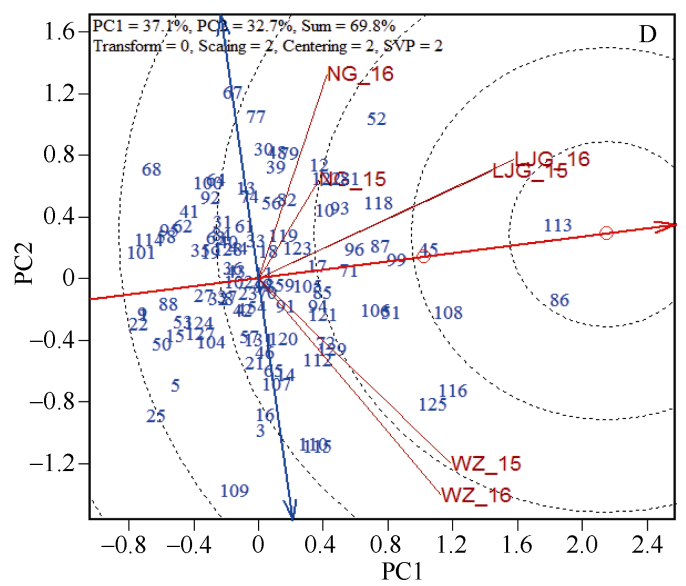

2016、五竹镇 2015、内官镇 2015、内官镇 2016 (图 3-C)。单株小薯产量 GGE 双标图中, 试点五竹镇 2016 最具鉴别力, 鲁家沟镇 2015 最具代表性, 综合 鉴别力和代表性, 试点排序依次为鲁家沟镇 2016、 鲁家沟镇 2015、五竹镇 2015、五竹镇 2016、内官 镇 2015、内官镇 2016 (图 3-F)。

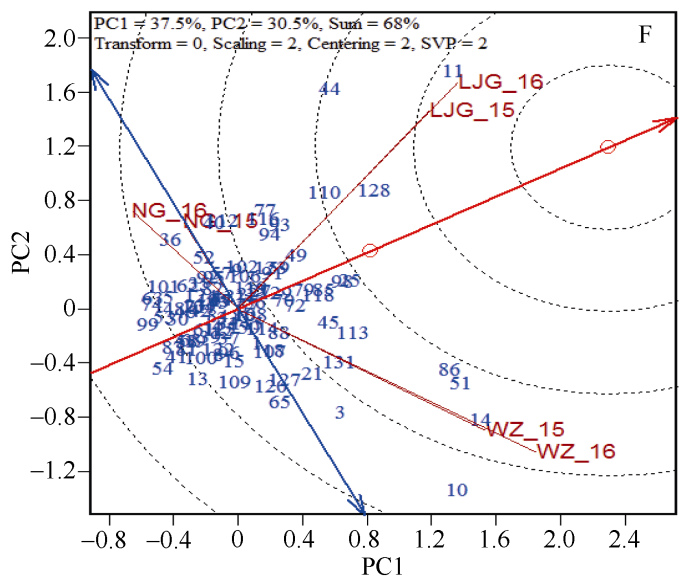

图 3 品系综合评价、环境代表性和鉴别力

Fig. 3 Lines ranking of comprehensive evaluation, environmental representativeness and the discriminating by the GGE biplot A：小区产量; B：小区大薯产量; C: 小区小薯产量; D：单株产量; E：单株大薯产量; F：单株小薯产量。

A: the yield of plot; B: the plot yield of large-sized tuber; C: the plot yield of small-sized tuber; D: the yield per plant; E: the yield of large-sized tubers per plant; F: the yield of small-sized tubers per plant. 


\section{3 讨论}

多数品种试验数据分析都是基于单一产量、品 质或抗性单个性状的数据, 但实际上在育种时需要 同时考虑多个性状。基于单一性状组分综合分析得 出的结论比单一性状分析的结论更重要 ${ }^{[23-24]}$ 。按照 马铃薯单一产量性状分析, 仅仅能够得出品系总产 量数据, 不能从总产量中获得大、小薯产量, 单株 大、小薯数及结薯数数据和影响这些数据的主要原 因 $^{[25-26]}$ 。

对 3 个试点马铃薯产量性状组分分析中发现, 小区总产量和大薯产量环境及互作效应中环境和互 作效应分别占总变异 $61.6 \%$ 和 50.8\%，其中互作效应 是影响小区总产量和大薯产量首要因素, 各参试品 系小区产量和大薯产量数据和图形相符; 小区小薯 产量中环境效应占总变异 $18.2 \%$, 环境是决定小区 小薯产量的首要因素。对小区总产量、小区大薯产 量与小区小薯产量决定因素不同, 所以小区产量组 分的 GGE 产量图中小区小薯产量图与前二者不相 符。环境效应是决定单株产量和单株大薯产量的首 要因素, 互作效应是影响单株小薯产量首要因素。 品系产量组分中结薯数是衡量产量的重要数据, 但 仅结薯数的数据不能完全说明结薯的整齐度和品系 大薯、小薯的分布程度。单株结薯数受环境效应影 响显著, 与单株大小薯产量相关的单株大薯数和单 株小薯数受互作效应影响显著。因基因型、环境、 基因型与环境互作对各产量组分决定程度有差异, 反映在产量适应性的双标图中, 多边形图形有相似 和不同。依照 GGE 产量适应性对参试品系的分析, 在 3 个试点适应性均强的品系有 G113、G106、G104 和 G116。青薯 9 号单株大薯产量同样受环境及互作 效应影响显著, 呈现出大薯产量不稳定。

品种试验中除需要评价每个产量性状中的组分 形成的原因, 还需要分析在多年品种试验中每个品 种产量组分的稳定性 ${ }^{[27-28]}$ 。对 2 年 3 个试点马铃薯 产量组分的稳定性分析发现, 小区总产量、小区大 薯产量的稳定性主要取决于环境和互作效应, 其中 环境效应是首要因素, 小区小薯产量稳定性取决于 互作效应。环境效应和互作效应是影响单株产量和 单株大小薯产量与单株小薯产量稳定性的主要因 素。本研究评估的所有产量组分中, 环境、基因型 与环境互作效应决定产量组分内稳定性的差异, 基 因型效应很小。依据产量性状的稳定性选择基因型 时, 试验地点的效应是显著的, 可以为特定的环境
选育稳定性差的高产基因型或者稳定性强的一般基 因型。试点环境对于所有产量组分都有显著影响时, 就需要选育出在不同环境中性状稳定性高的基因 型。按小区产量来选择, 可将所有基因型可以分为 三类。第一类是具有高产稳定性基因型，如 G124、

G125、G122、G10、G107、G25、青薯 9 号，这类 基因型可适应于不同生态环境。第二类是高产不稳 定性基因型，如 G86、G94、G27、G65、G16、G79、 $\mathrm{G} 3$, 这类基因型可适于特定的环境。第三类基因型 是低产稳定性的基因型，如 G64、G82、G39、G4、 G23、G22、G95，这类基因型可用于除产量以外的 品质性状选择。广适高产稳定性强的基因型在各试 点都体现出基因型主效显著, 互作效应显著时表现 为环境对基因型有利。广适高产稳定性差基因型在 各试点的基因型主效差异显著, 不同基因型受互作 效影响差异同样显著, 这类在特定生态区表现出高 产稳定性的品种是主要选择对象。篮选特定生态区 高产稳定性强的基因型是更有效的品种稳定性选择 方法。

试点评价的目的是鉴别试点是否适合品种试验, 核心试点应该对目标环境具有高度代表性，同时对 基因型的差异具有很强的鉴别力 ${ }^{[23]}$ 。如何判断品种 试验设置的试点是否是同一生态下的最佳试点是提 高品种选择效率的重要工作。试点 GGE 双标图能够 将年份和试点分开展示, 在 3 个试点鉴别各基因型, 所有产量组分在 2 个年份中鲁家沟镇和五竹镇表现 一致，环境和基因型互作效应在年份间不显著。各 基因型小区小薯产量在试点内官镇的 2 个年份间差 异显著, 原因是年份间的气候变化。产量组分最高 的基因型因试点不同存在差异，根据试点年份的组 合环境划分为不同的品种生态区，环境分组与试点 气候条件相符, 3 个试点中内官镇和鲁家沟镇同属典 型的干旱和半干旱生态区, 而五竹镇属高寒阴湿 区。同属干旱半干旱区的 2 个试点中鲁家沟镇对目 标生态区域的代表性强于内官镇, 但在 2 个生态区 中, 高寒阴湿区的试点五竹镇比干旱半干旱区试点 鲁家沟镇和内官镇有鉴别力。

\section{4 结论}

GGE 模型对产量各组分的分析可以综合评价并 篮选出优良品系。本研究篮选出的高产稳定品系有 G116、G124、G125、G122 和青薯 9 号, 高产不稳 定的品系有 G86、G10、G121、G106、G107 和 G72。 最理想的生态区试点是鲁家沟镇，对品种的鉴别力 


\section{最强的试点是渭源五竹镇。}

\section{References}

[1] Zaheer K, Akhta M H. Potato production, usage, and nutrition-a review. Crit Rev Food Sci, 2014, 56: 711-721.

[2] 王玉萍, 隋景航, 梁延超, 卢潚. 甘肃省两个生态区马铃薯加 工品质差异和加工品系篎选. 甘肃农业大学学报, 2016, 51(5): 39-45.

Wang Y P, Sui J H, Liang Y C, Lu X. Screening for potato processing lines with tuber qulity index from two ecoregions. $J$ Gansu Agric Univ, 2016, 51(5): 39-45 (in Chinese with English abstract).

[3] Mulema J M K, Adipala E, Olanya O M. Yield stability analysis of late blight resistant potato selections. Crop Sci, 2016, 56: 1645-1661.

[4] Padi F K. Relationship between stress tolerance and grain yield stability in cowpea. J Agric Sci, 2004, 142: 431-443.

[5] Bednarz C W, Bridges D C, Brown S M. Analysis of cotton yield stability across population densities. Semigroup Forum, 2000, 92: 128-135.

[6] Fan X M, Kang M S, Chen H M. Yield stability of maize hybrids evaluated in multi-environment trials in Yunnan, China. Agron J, 2007, 99: 220-228.

[7] Mekbib F. Yield stability in common bean (Phaseolus vulgaris L.) genotypes. Euphytica, 2003, 130: 147-153.

[8] Aastveit A H, Martens H. ANOVA interactions interpreted by partial least squares regression. Biometrics, 1986, 42: 829-844.

[9] Eberhart S A, Russel W A. Stability parameters for comparing varieties. Crop Sci, 1966, 6: 36-40.

[10] Blouin D C, Webster E P, Bond J A. On the analysis of combined experiments. Weed Technol, 2015, 25: 165-169.

[11] Kang M S. Simultaneous Selection for yield and stability in crop performance trials: consequences for growers. Agron J, 1993, 85: 754-757.

[12] Gauch H G. Statistical analysis of yield trials by AMMI and GGE. Crop Sci, 2006, 46: 1488-1500.

[13] Yan W K, Fetch J M, Fregeau-reid J. Genotype × location interaction patterns and testing strategies for oat in the Canadian. Prairies, 2011, 51: 1903-1914.

[14] Yan W K, Kang M S, Ma B L. GGE biplot vs. AMMI analysis of genotype-by-environment data. Crop Sci, 2007, 47: 641-653.

[15] Nzuve F, Githiri S, Mulunya D M. Analysis of genotype $\times$ environment interaction for grain yield in maize hybrids. J Agric Sci, 2013, 5: 75-85.

[16] Soto B J, Duquid S, Booker H. Genomic regions underlying agronomic traits in linseed (Linum usitatissimum L.) as revealed by association mapping. J Integr Plant Biol, 2014, 56: 75-87.

[17] Murphy S E, Lee E A, Woodrow L. Genotype $\times$ environment interaction and stability for is oflavone content in Soybean. Crop
Sci, 2009, 49: 1313-1321.

[18] Wachira F, Ngetich W, Omolo J. Genotype $\times$ environment interactions for tea yields. Euphytica, 2002, 127: 289-297.

[19] Burgueño J, Campos G D L, Weigel K. Genomic prediction of breeding values when modeling genotype $\times$ environment interaction using pedigree and dense molecular markers. Crop Sci, 2012, 52: 707-712.

[20] Phuke R M, Anuradha K, Radhika K, Jabeen F, Anuradha G, Ramesh T, Hariprasanna K, Mehtre S P, Deshpande S P, Anil G, Das R R, Rathore A, Hash T, Reddy B V S, Kumar A A. Genotype $\times$ environment interaction, correlation, and GGE Biplot analysis for grain Iron and zinc concentration and other agronomic traits in RIL population of sorghum (Sorghum bicolor L. Moench). Front Plant Sci, 2017, 5: 712-716.

[21] Zolfaghar S, Bahram H, Dadkhodaie A. Dissection of genotype $\times$ environment interactions for mucilage and seed yield in Plantago species: application of AMMI and GGE biplot analyses. PLoS One, 2018, 13: e0196095.

[22] 张永成, 田丰. 马铃薯试验研究方法. 北京: 中国农业科学技 术出版社, 2007. pp 90-93.

Zhang Y C, Tian F. Potato Experiment Test Research Method. Beijing: China Agriculture Science and Technology Press, 2007. pp 90-93 (in Chinese).

[23] 严威凯. 农作物品种试验数据管理与分析. 北京: 中国农业科 学技术出版社, 2015. pp 133-146.

Yan W K. Crop Variety Trials Data Management and Analysis. Beijing: China Agriculture Science and Technology Press, 2015. pp 133-146 (in Chinese).

[24] Dia M, Weherr T C, Hasssel R, Price D S. Genotype × environment interaction and stability analysis for watermelon fruit yield in the United States. Crop Sci, 2016, 56: 1645-1661.

[25] Wang R H, Hu D H, Zheng H Q, Yun S. Genotype × environmental interaction by AMMI and GGE biplot analysis for the provenances of Michelia chapensis in South China. J For Res, 2016, 27: 659-664.

[26] Muthoni J, Shimelis H, Melis R. Genotype $\times$ environment interaction and stability of potato tuber yield and bacterial wilt resistance in Kenya Am J Potato Res, 2015, 92: 367-378.

[27] 严威凯. 双标图分析在农作物品种多点试验中的应用. 作物 学报, 2010,36: 1805-1819.

Yan W K. Optimal use of biplots in analysis of multi-location variety test data. Acta Agron Sin, 2010, 36: 1805-1819(in Chinese with English abstract).

[28] 严威凯, 盛庆来, 胡跃高, Hunt L A. GGE 叠图法: 分析品种火 环境互作模式的理想方法. 作物学报, 2001, 27: 21-28.

Yan W K, Sheng Q L, Hu Y G, Hunt L A. GGE Biplot: an ideal tool for studying genotype by environment interaction of regional yield trial data. Acta Agron Sin, 2001, 27: 21-28 (in Chinese with English abstract). 